\title{
tudor, a posterior-group gene of Drosophila melanogaster, encodes a novel protein and an mRNA localized during mid-oogenesis
}

\author{
George S. Golumbeski, Anne Bardsley, Franciscus Tax, and Robert E. Boswell ${ }^{1}$ \\ Department of Molecular, Cellular, and Developmental Biology, University of Colorado, Boulder, Colorado 80309 USA
}

\begin{abstract}
The tudor (tud) locus of Drosophila melanogaster is required during oogenesis for the formation of primordial germ cells and for normal abdominal segmentation. The tud locus was cloned, and its product was identified by Northern analysis of wild-type and tud mutant RNAs. The locus encodes a single mRNA of $\sim 8.0 \mathrm{~kb}$ that is expressed throughout the life cycle, beginning in the early stages of germ-line development in the female. During oogenesis, tud mRNA appears to be present in the oocyte precursor within the germarial cysts, and in stages 1-3 it accumulates within the developing oocyte. The transcript is localized to the posterior half of the oocyte during oogenetic stages 4-7 but is not detectable within the ooplasm by egg deposition and throughout early embryogenesis. The tud protein has a predicted molecular mass of 285,000 daltons and has no distinctive sequence similarity to known proteins or protein structural motifs. Taken together, these results indicate that the tud product is a novel protein required during oogenesis for establishment of a functional center of morphogenetic activity in the posterior tip of the Drosophila embryo.
\end{abstract}

[Key Words: Drosophila melanogaster; tudor; oogenesis; pattern formation]

Received May 14, 1991, revised version accepted August 27, 1991.

Elucidation of the molecular mechanisms by which pattern formation is initiated within the early embryo remains one of the most difficult problems in developmental biology. However, classical and recent evidence suggest that in many organisms early determinative events are initiated by the asymmetric distribution of maternally encoded cytoplasmic components (Davidson 1986). In the Drosophila melanogaster embryo, at least two different kinds of determinative asymmetries have been identified: localized determinants that are required for germ-cell determination (Boswell and Mahowald 1985a), and morphogenetic gradients of informational molecules that are involved in organizing the anteroposterior (Nüsslein-Volhard et al. 1987; Manseau and Schüpbach 1989a), and the dorsoventral body axes of the early embryo (Anderson 1987; Roth et al. 1989; Rushlow et al. 1989; Steward 1989).

The initiation of germ-line development in insects and anuran amphibians represents one of the most extensively studied examples of cytoplasmically localized information specifying cell fates (Boswell and Mahowald 1985a). In Drosophila (at $25^{\circ} \mathrm{C}$ ), the pole cells, or primordial germ cells, form at the posterior tip of the embryo at $\sim 90 \mathrm{~min}$ of development. Because the other cleavage-

\footnotetext{
${ }^{1}$ Corresponding author.
}

stage nuclei do not cellularize until $3 \mathrm{hr}$ of development, the pole cells are the first cell type to differentiate in the early embryo. During gastrulation, the pole cells are carried to the embryonic gonads, where they ultimately become the germ cells within the ovaries and testes.

Initially, the pole cells arise from a region of the egg marked by distinctly localized cytoplasmic organelles. In fact, these organelles, the polar granules, are the only ooplasmic organelles known to be localized within the early embryo. These structures are present at the posterior pole of the egg in a distinctly clear region of ooplasm, the germ plasm, and are incorporated into the pole cells as they form. Ultrastructural and biochemical analyses of the germ plasm have shown that the polar granules lack delimiting membranes and consist of RNA and protein (Eddy 1975; Mahowald 1977). Furthermore, similar structures are associated with the primordial germ cells and/or germ plasm in a wide variety of divergent species: many dipterans (Boswell and Mahowald 1985a), Caenorhabditis elegans (Strome and Wood 1981), and Xenopus laevis (Smith and Williams 1975).

In Drosophila, the germ plasm is capable of inducing the ectopic formation of histologically discernible pole cells when transplanted to ectopic sites within the preblastoderm embryo (Illmensee and Mahowald 1974, 1976). By transplanting posterior pole plasm from oocytes at various stages of oogenesis, Illmensee et al. 
(1976) demonstrated that this instructive activity was fully functional by stage 13 of oogenesis (for review of oogenesis, see King 1970). However, cytoplasm transplanted from other regions of the egg or early embryo does not induce pole-cell formation. Transplantation of the ectopically induced pole cells into genetically marked recipient embryos demonstrated that these cells are capable of forming functional germ cells. Thus, before the completion of oogenesis, the germ plasm of Drosophila contains a localized factor or factors capable of autonomously initiating the formation of the germline.

In addition to its role in germ-cell determination, the posterior pole plasm is necessary for the specification of the anteroposterior body axis of the Drosophila embryo (Frohnhöfer et al. 1986). When posterior pole plasm is removed from preblastoderm embryos, the embryos develop lacking abdominal segments. Similarly, removal of cytoplasm from the anterior pole results in the absence or significant reduction of head structures in the resulting embryos. In contrast, removal of cytoplasm from egg regions other than the poles produces only local defects. Thus, in the mature egg, the two poles appear to contain centers of morphogenetic activity with long-range effects on pattern formation. Moreover, both the anterior and posterior polar plasms can reorganize pattern and polarity when transplanted to ectopic regions within the egg. Thus, the components present at each pole appear to have the instructive properties expected of morphogens.

Extensive genetic analyses have revealed that each organizing center depends upon the proper function of several maternal-effect loci (Anderson 1987; Nüsslein-Volhard et al. 1987; Manseau and Schüpbach 1989a). At least three genes, bicoid, exuperantia, and swallow, are necessary for the normal functioning of the anterior center. For example, embryos from homozygous bicoid females lack head and thoracic structures, display an extended abdominal region, and may contain a second telson at the anterior pole. However, a normal anterior pattern can be restored in the mutant embryos if cytoplasm from the anterior tip of wild-type eggs, or bicoid RNA, is transplanted to the anterior tip of the mutant embryos (Frohnhöfer and Nüsslein-Volhard 1986; Driever et al. 1990). In comparison, the posterior organizing center appears to be more complex. Currently, at least 10 genes are known to be required for normal organization of the posterior center (cappucino, mago nashi, nanos, oskar, pumilio, spire, staufen, tudor, valois, and vasa; hereafter referred to as the posterior group) (Boswell and Mahowald 1985b; Lehmann and NüssleinVolhard 1986; Schüpbach and Wieschaus 1986; Lehmann and Nüsslein-Volhard 1987; Manseau and Schüpbach 1989b; Boswell et al. 1991). Embryos from homozygous pumilio or nanos females lack abdominal segments, and embryos from females with mutations in any of the other posterior group genes display abdominal segmentation defects and fail to form germ cells. In all posterior group mutants tested, the abdominal segmentation defect can be rescued by transplantation of wildtype posterior pole plasm into the prospective abdominal region of the mutant embryos. In addition, on the basis of studies with oskar, transplantation of wild-type pole plasm can rescue the defect in pole cell formation only when transplanted directly into the posterior tip of the mutant embryos (Lehmann and Nüsslein-Volhard 1986). Thus, genetic analyses and transplantation studies indicate that the posterior pole plasm is required both for germ-cell determination and for proper establishment of the abdominal body pattern. Whether the germ plasm and the posterior organizing center are the same, different, or interdependent entities remains unclear.

Despite the identification of many of the components of the morphogenetic centers, a detailed understanding of these early determinative processes requires, $(1)$ an identification of the molecular nature of the gene products required for germ-cell determination and anteroposterior patterning, (2) an analysis of how these molecules act and interact to initiate developmental commitments, and (3) an understanding of how these activities become properly distributed within the ooplasm.

We have concentrated on identifying and analyzing genes required for the proper function of the posterior organizing center. One of these genes, tudor, (tud), is required for both abdominal segmentation and pole-cell determination (Boswell and Mahowald 1985b). Here, we describe our initial molecular analysis of the tudor locus. The gene has been cloned and sequenced, and the spatial and temporal distribution of tud mRNA during Drosophila development has been determined. Our results demonstrate that the tud mRNA accumulates within the posterior region of the developing oocyte during the early to middle stages of oogenesis. In addition, the tudor locus encodes an unusually large protein with no functional counterparts in the current data bases. Thus, the tud gene product is a novel protein that is most likely required during early oogenesis for establishment of a functional posterior organizing center and/or germ plasm.

\section{Results}

Cloning and cytological localization of tud

Previous recombination and complementation analyses placed the tud locus within $D f(2 R) P F 1$, very close, and distal to the Punch (Pu) locus (O'Donnell et al. 1989). Thus, a chromosomal walk was undertaken to clone the tud region from an entry point within $57 \mathrm{C}$ ( $\lambda$ clone 525 ; Levy et al 1982). In all, $\sim 200 \mathrm{~kb}(57 \mathrm{C} 3-57 \mathrm{C} 8)$ of genomic DNA was isolated from recombinant genomic libraries in $\lambda$ Charon 4A and EMBL4 (Maniatis et al. 1978; McLean et al. 1990).

${ }^{3} \mathrm{H}$-Labeled recombinant phage from the chromosomal walk were then hybridized to polytene chromosomes from $t u d^{5}$ and $t u d^{B 46}$ mutants (these alleles are associated with inversion breakpoints that disrupt $t u d^{+}$function). This analysis revealed that clone $\mathrm{T} 1$ spanned the tud $d^{5}$ breakpoint (shown in Fig. 1B) and that the 525 clone spanned $P u$ inversions, as well as the $t u d^{\mathrm{B} 46}$ inversion (data not shown). 
Figure 1. In situ hybridization of tud probes to polytene chromosomes from tud $d^{5} /$ tud $^{5}$ larvae. $(A-D)$ Chromosome $2 R$. The arrowheads identify polytene chromosome interval 58. Arrows indicate the site of labeling of the probe. The $t u d^{5}$ mutation is an inversion between the 46F8-10 region and the 57C6-9 region of chromosome $2 R$. (A) Genomic clone $\mathrm{Tl}$ was ${ }^{3} \mathrm{H}$-Labeled and hybridized to wild-type chromosomes. Labeling is indicated by the arrow in region 57. $(B)^{3} \mathrm{H}$-Labeled $\mathrm{Tl}$ probe was hybridized to $t u d^{5}$ chromosomes. Labeling is observed on either side of the inversion. $(C)$ cDNA clone ST3 was ${ }^{3} \mathrm{H}$-labeled and hybridized to $t u d^{5}$ chromosomes. The site of labeling is adjacent to the distal inversion breakpoint. (D) cDNA clone ST58 was ${ }^{3} \mathrm{H}-\mathrm{la}$ beled and hybridized to $t u d^{5}$ chromosomes. The site of labeling is adjacent to the proximal inversion breakpoint. (Insert) A higher magnification view of the site.

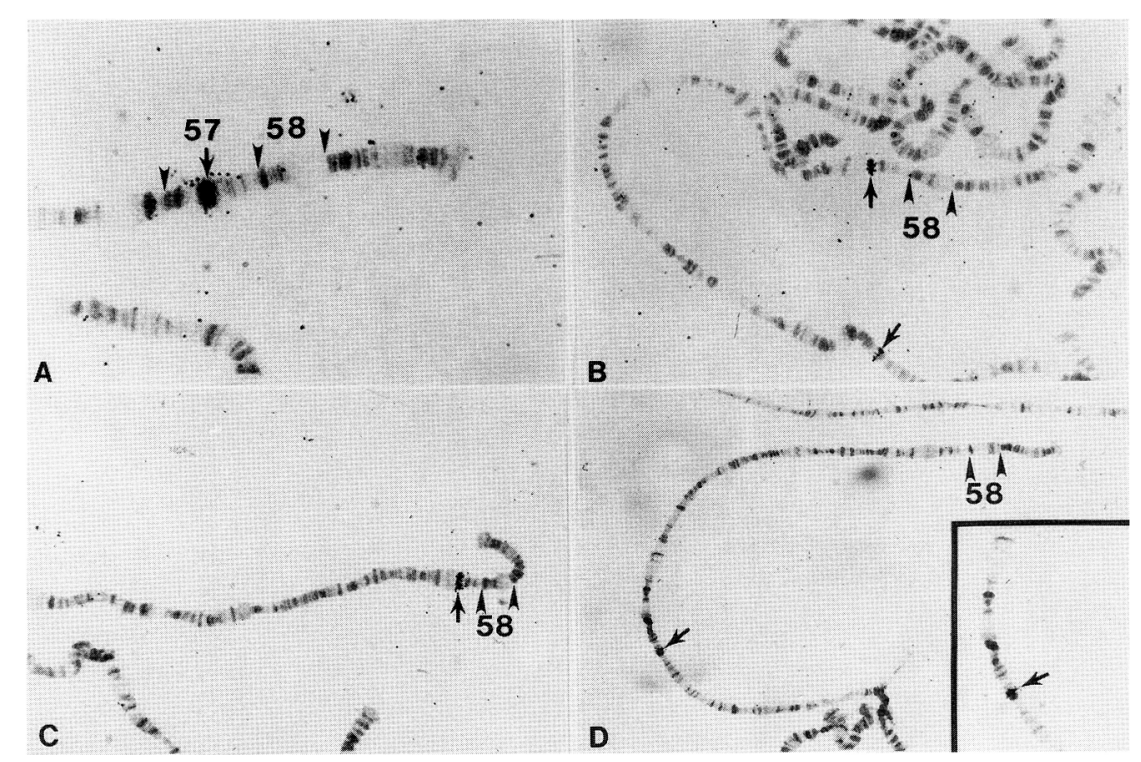

On the basis of these data, the tud locus was tentatively mapped to the 525-T1-T2 region. A map of this region is presented in Figure 2. As illustrated, a cosmid clone $\left(\cos \mathrm{H}_{\text {; }}\right.$ isolated on the basis of its sequence similarity to 525-T1-T2) that spanned the cloned region most likely to contain tud was isolated. Northern analysis of poly $(\mathrm{A})^{+}$RNA from 0 - to 4-hr embryos revealed six transcripts homologous to $\cos \mathrm{H}$. Of these six transcripts, five were derived from the most proximal end of $\operatorname{cosH}$, which corresponds to the Punch region, or a region proximal to $P u$ (data not shown). The remaining transcript ( $8 \mathrm{~kb}$ in length) was derived from a region distal to
$\mathrm{Pu}$. Overlapping recombinant phage clones extending $\sim 30 \mathrm{~kb}$ past the distal end of $\cos \mathrm{H}$ were used to probe for other early embryonic transcripts, but none were found. To directly verify the identity of the $8-\mathrm{kb}$ transcript, Northern analysis was performed on poly $(\mathrm{A})^{+} \mathrm{RNA}$ isolated from embryos derived from $t u d^{2}$ and $t u d^{5}$ homozygous females. As presented in Figure 3, A and B, in each mutant the size of the 8-kb transcript was altered; however, no other transcript homologous to $\cos \mathrm{H}$ was altered (data not shown). The 8-kb RNA is expressed preferentially in adult females, and this RNA is abundant and localized within the posterior ooplasm of developing
Figure 2. A molecular map of the tudor region. CDNA clones discussed in the text (ST3, ST58, 9A1, and JO) are represented as broad, stippled lines shown above the distance scale. The proximal and distal ends of cosmid $\mathrm{H}$ (shown above the four cDNA clones) are $\sim 11 \mathrm{~kb}$ beyond 0 and 5 $\mathrm{kb}$ beyond 24 on the distance scale, respectively. Approximate physical limits of the tud locus and the direction of tud transcription are depicted on the map of cosmid H. Genomic phage clones recovered in the chromosomal walk (T2, T1, and 525) are shown above cosmid H. Each of these clones ends in a synthetic EcoRI restriction site. (E) EcoRI; (K) KpnI; (S) SalI; (Sc) SacI; (X) XhoI.
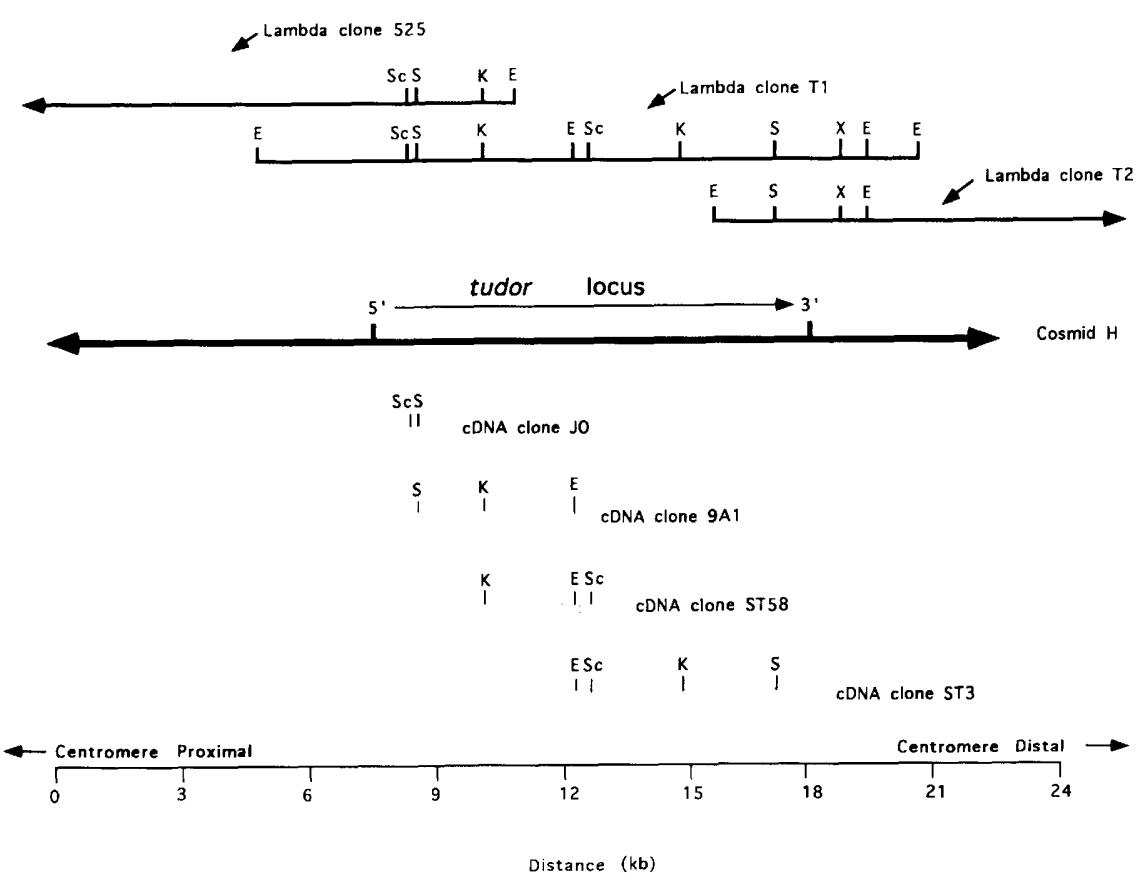

Distance (kb) 


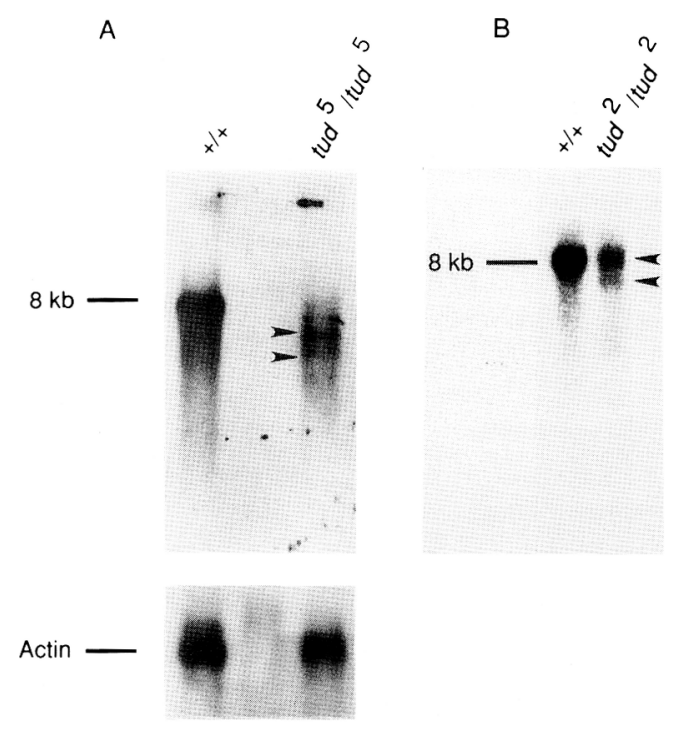

C

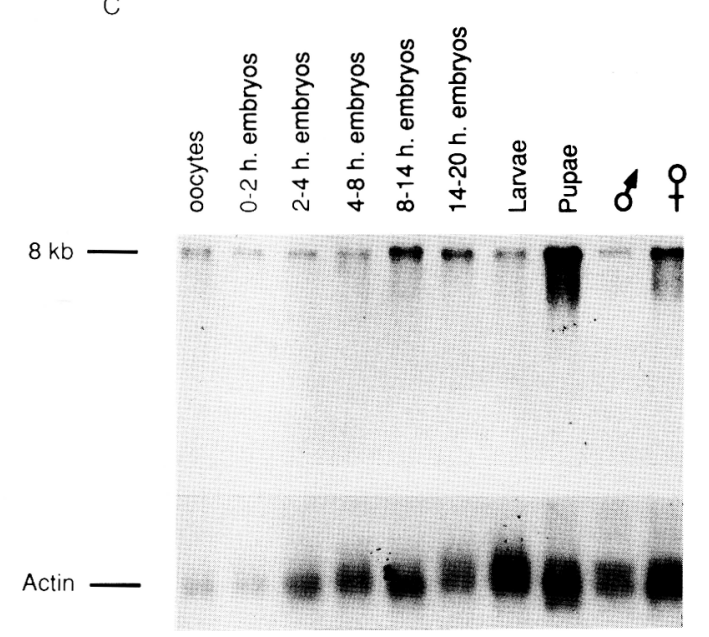

Figure 3. Northern analysis of tud transcription. All Northern blots were probed with ${ }^{32}$ P-labeled tud cDNA clone ST58. $\mid A$ and $B)$ Changes in the tud transcript in tud mutant embryos. $(A)$ Five micrograms of poly $(\mathrm{A})^{+}$RNA from 0 - to 6-hr embryos derived from mothers of the indicated genotypes was hybridized with the tud probe. A cDNA clone of Drosophila actin (Act5c; Fyrberg et al. 1980; Tobin et al. 1980) was used as a control for quality of the RNA. Note the two novel species of tud-homologous RNA produced by $t u d^{5}$ embryos (arrowheads). (B) Similar Northern blot showing a novel transcript produced by embryos derived from $t u d^{2}$ mothers (indicated by the lower arrowhead). The filter was stripped and reprobed with the cosmid clone $\cos \mathrm{H}$, which encompasses the tud trancription unit and also hybridizes to several other trancripts. The $8-\mathrm{kb}$ tud transcript was the only transcript altered in any of the tud mutants (data not shown). (C) Developmental profile of tud RNA expression. Lanes contain $\sim 500 \mathrm{ng}$ of poly $(\mathrm{A})^{+}$RNA from flies at the indicated stages of development. Oocytes denotes RNA prepared from mature unfertilized eggs (oogenetic stage 14). (0-2h., 2-4h., 4-8h., 8-14h.. and 14-20h.). RNA prepared from embryos of the indicated period (hour) of embryogenesis. Larvae and pupae denote RNA from a mixture of larval stages and pupal stages, respectively. Male and female symbols denote RNA from adults of the indicated sex. The $A c t 5 c$ probe was used as a control for quality of the RNA. oocytes (see below). This expression pattern is completely consistent with the genetic analysis of the tud locus (Boswell and Mahowald 1985b; O'Donnell et al. 1989|. Taken together, these results demonstrate that the $8-\mathrm{kb}$ poly $(\mathrm{A})^{+}$RNA is a product of the tud locus. We have no evidence suggesting that the tud locus encodes any additional RNAs.

\section{Isolation and characterization of tud $c D N A$ clones}

We screened $>1.25$ million unamplified, recombinant phage from a Drosophila embryonic $(0-24 \mathrm{hr})$ cDNA library and isolated $18 \mathrm{cDNAs}$ that hybridize to the tud transcript. Restriction endonuclease mapping and Southern analysis of these clones revealed that ST3 contains a 4.5-kb cDNA insert that extends from the $3^{\prime}$ end of the tud locus to the middle of the gene. ST58 contains a $2.5-\mathrm{kb}$ insert that extends from the middle of the tud gene toward, but not to, the $5^{\prime}$ end of the locus. A third clone, JO (kindly provided by J. O'Donnell), contains a 1.0-kb cDNA that extends from the $5^{\prime}$ end of tud. Because ST3 and ST58 contain $\sim 600$ bp of overlap, ST3, ST58, and JO, together, represent $\sim 7.3 \mathrm{~kb}$ of the $8-\mathrm{kb}$ tud transcript (Fig. 2). To recover the remaining fragment of tud cDNA, we screened a $D$. melanogaster cDNA library obtained from Stratagene using probes that flanked the missing tud sequence. This screen resulted in the recovery of the cDNA clone 9Al, which contains a $3.0-\mathrm{kb}$ insert and represents the remainder of the tud transcript (Fig. 2).

To further verify that these cDNA clones are derived from the tud locus, each of the four cDNA inserts was labeled with ${ }^{3} \mathrm{H}$-labeled dATP or with digoxigenin-modified dUTP and hybridized to polytene chromosomes from wild-type and $t u d^{5}$ larvae. All of the clones hybridized to the presumptive tud region (57C7-57C8). Moreover, ST3 and ST58 were found to lie on opposite sides of the $t u d^{5}$ inversion (see Fig. 1C,D). Thus, these cDNAs are products of the tud locus.

\section{Sequence analysis of the tud gene product}

We determined the complete double-stranded sequence for JO, ST58, and ST3. Furthermore, we determined the double-stranded sequence for the genomic DNA comprising the gap between JO and ST58. This sequence was then confirmed by sequencing one strand of the corresponding cDNA clone, 9A1. We also sequenced genomic DNA containing the single EcoRI restriction endonuclease site, which represents the junction between ST58 and ST3, to verify that no additional tud sequence was present between these clones. Furthermore, sequence analysis of genomic DNA containing the $3^{\prime}$ end of the tud transcript revealed that the 16 deoxyadenosine monophosphate residues present at the $3^{\prime}$ end of ST3 are not present in the genomic sequence (data not shown). Finally, primer extension studies demonstrated that the $5^{\prime}$ end of the JO cDNA is within 50 bases of the $5^{\prime}$ end of the tud transcript (data not shown).

By correlating the restriction map of the genomic 
DNA comprising the tud locus with the restriction map of the complete tud cDNA, we determined that the tud transcription unit is $\sim 8.8 \mathrm{~kb}$. On the basis of the combined length of the four cDNA clones, this transcription unit encodes a poly $(\mathrm{A})^{+}$RNA of at least 8054 nucleotides. As shown in Figure 4, our analysis of the tud sequence revealed that the tud transcript can encode a polypeptide of 2515 amino acids that has a molecular mass of $285 \mathrm{kD}$. The first methionine within the predicted open reading frame (ORF) (position 219) matches the initiation methionine consensus sequence for Drosophila (Cavener 1987). This ORF extends to position 7782 where a UGA codon occurs. A search of current sequence data bases revealed that the predicted amino acid sequence of tud shows no significant similarity to any polypeptide sequences previously reported. The tud polypeptide contains two potential tyrosine kinase phosphorylation sites (amino acid positions 1369 and 1409) and 24 potential amino-glycosylation sites. The amino acid composition of the tud protein is similar to the average compiled for Drosophila proteins by M. Ashburner and reported by Smoller et al. (1990). Thus, the tud protein is a novel polypeptide with no apparent counterparts in the available data bases.

\section{Expression of the tud gene}

To determine the pattern of tud transcription during development, poly $(\mathrm{A})^{+}$RNA was prepared from various stages of the Drosophila life cycle. These RNAs were fractionated on agarose gels, transferred to nylon membranes, and probed with ${ }^{32} \mathrm{P}$-labeled tud cDNAs. The results, shown in Figure 3, reveal that the tud locus encodes a single 8 -kb transcript throughout the life cycle and that this message is present at all developmental stages. While the early expression of tud (during oogenesis and early embryogenesis) was expected from earlier genetic analyses, its presence during the later stages of embryogenesis, larval, and pupal life was not predicted by genetic studies. The existing tud alleles have no apparent effect on larval, pupal, or adult viability (Boswell and Mahowald 1985b).

\section{Localization of the tud transcript}

To gain further insight into the function of the tud gene product, we studied the distribution of tud mRNA during oogenesis. In Drosophila, oogenesis is divided into 14 stages, beginning with stage 1, a 16-cell cluster at the

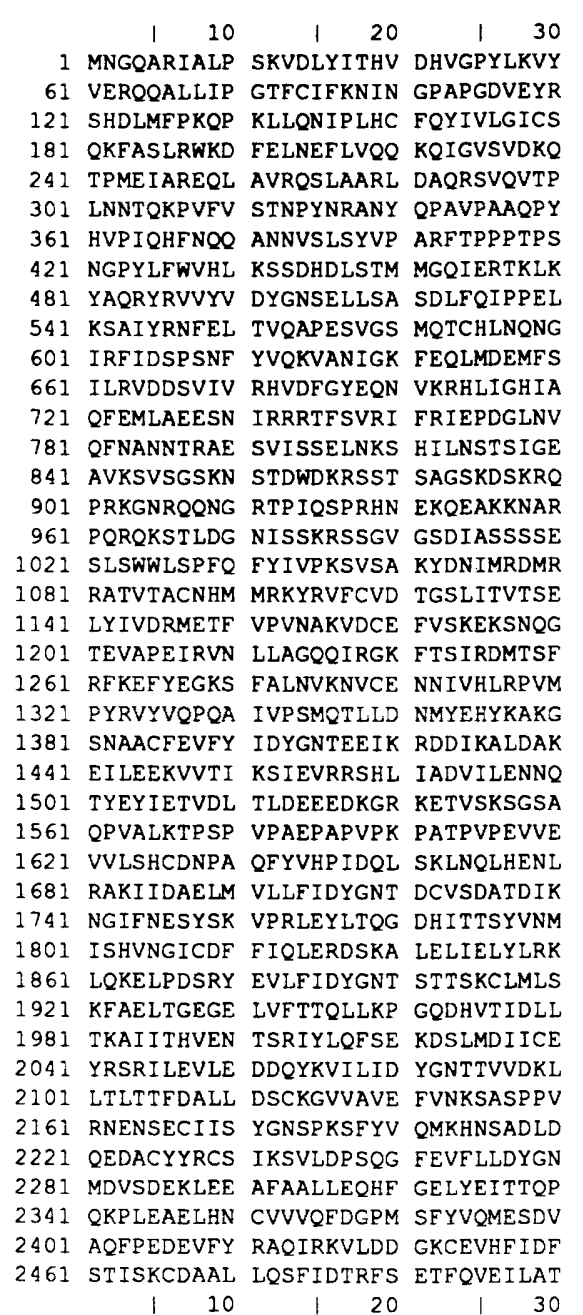

\begin{tabular}{|c|c|c|c|}
\hline 40 & 50 & 60 & \\
\hline & & AIEPSWS & . \\
\hline & & VDFGYNRTVD & 120 \\
\hline QTDLAEV & RRLVVNQIVK & ITVEPTQICD & 180 \\
\hline DHCKKLK & KDNPQSPVTE & YNNNSIHNSK & 240 \\
\hline & YTPKHLPLVN & VTNVQVQMPG & 300 \\
\hline ANPRSQY & TYYNVRMNKP & INAMPPPAGP & 360 \\
\hline HQIPIPA & FRTTSLTVGL & TYDVVISYVE & 420 \\
\hline ZAPELGT & ACVARFSEDG & HLYRAMVCAV & 480 \\
\hline KPFAFRE & ALAGTKEIEP & IDDSMKRIFK & 540 \\
\hline MLELLRQI & KNSRQSYKKA & EQLENDDAVE & 600 \\
\hline ATREFPD & QLILGAPCIV & KCDQEWYRAE & 660 \\
\hline EMPRQA & IKCCLKGFEN & SELSEDKITD & 720 \\
\hline LAKNLNV & MKKLYKLLSMP & FEQYLSLEKG & 780 \\
\hline RLQEQEF & EQQOKKVDVR & QQQLAVEIPQ & 840 \\
\hline$D F$ & HLDFSCETQS & TGSYSSGMSS & \\
\hline NSESPRRS & RDGQQGNQRS & QNAPQGYAQK & 960 \\
\hline AAKPEKY & VSLDKPYALQ & EMKTPSKEAA & 1020 \\
\hline KQHQP & LQLI & VRQ & 1080 \\
\hline QLEQRFA & DPPCMAHRCS & FHSVVTNYDP & 1140 \\
\hline SSTCSYT & VNIFVNGASL & RDMLVKAEFL & 1200 \\
\hline FDYGNNV & NFLCTYDDAK & FVKSNPNLAR & 1260 \\
\hline AEDRRSF & ICP & VYTAK & 1320 \\
\hline KKFDVGQ & RSSDGN & WYRARISGKD & 1380 \\
\hline HASGFAV & EINLPIGRPS & NDTKLKARIS & 1440 \\
\hline$K$ & LVF & KGKSR & 15 \\
\hline KKOHN & DKD & PAEPARTVAP & 1560 \\
\hline TVRE & KQAPA & YKDLDC & 1620 \\
\hline SLPQI & MNVVNGADCV & SMYSVDKCWY & 1680 \\
\hline HIEPE & CLPCALPIRP & KGTADWVDAA & 1740 \\
\hline DVAKK & GFARPL & EYLASGCSCY & 1800 \\
\hline LKP LEGF & EKGLIVAALF & EDDELWYRAQ & 1860 \\
\hline PSLS & KKCSLQLP & YISWSPEAEA & \\
\hline I IDF & KEP & EASKESLAVT & 1980 \\
\hline GSKLQPK & TEKAAVDDMC & VVQFADDLEF & 2040 \\
\hline EFTI & KPVAEICSM & PSAIFEKNK & \\
\hline & $\mathrm{LF}$ & KLIQK & 2160 \\
\hline TLQSLK & KEKLKKLIDP & TTNSNGVCYS & 2220 \\
\hline VWS & & ALHCQLSKIP & 2280 \\
\hline & & $\operatorname{TGV}$ & 2340 \\
\hline zint & LDAEQDLPAF & SDLKEGALCV & 2400 \\
\hline & QLPEELAK & RYSRHCELDF & 2460 \\
\hline 18 & CQSKN I & LQECQ & 2515 \\
\hline
\end{tabular}

tein encoded by the tud locus. 


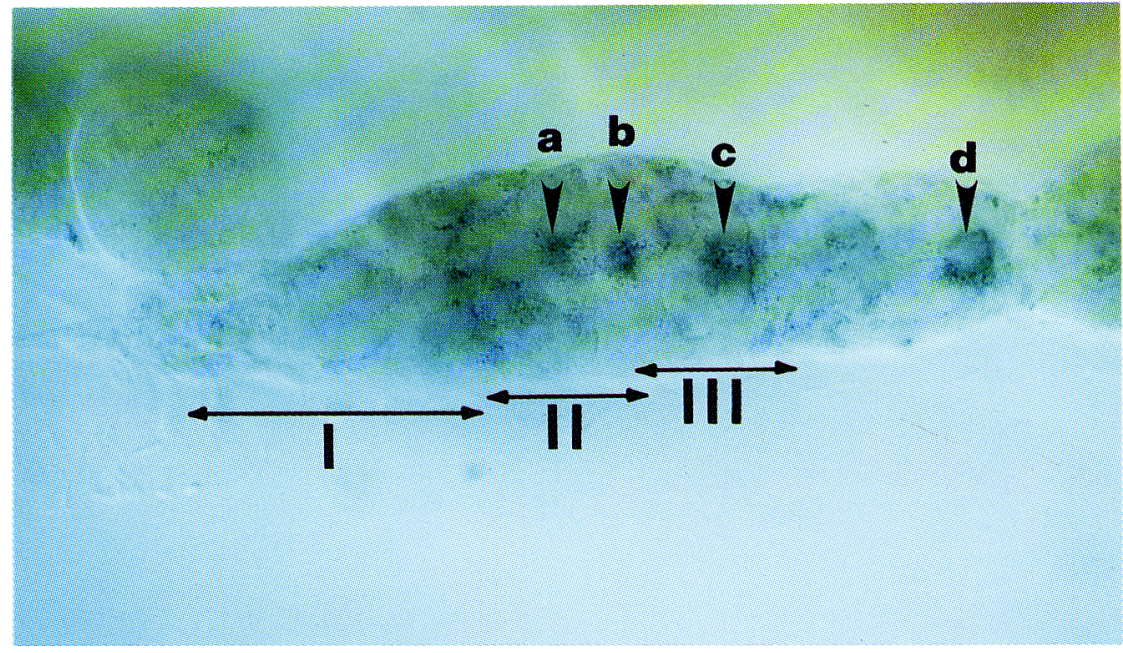

Figure 5. Localization of the tud transcript in the Drosophila germarium by in situ hybridization. Germarium from a wild-type ovariole (whole mount) hybridized with digoxigenin-labeled tud cDNA probe ST58. The anterior portion of the germarium is to the left, and a stage egg chamber is emerging from the germarium at its posterior end (right). Germarial regions $1-3$ are indicated by arrows marked by roman numerals. Arrowhead indicate cystocytes stained with tud probe. $\mid a$ and b) Cystocytes within cysts in region $2 ;(c)$, stained cystocyte occupying a posterior position in a region $3 \mathrm{cyst}$ (d) the perinuclear localization of tud transcript within the oocyte of a stage 1 egg chamber.

terminus of the germarium, and ending at stage 14 with a fully mature oocyte. The germarium occupies the distal region of the ovariole and contains the primary oogonia, or stem cells. Each stem cell division produces one stem cell and one daughter cystoblast, which will divide four times to produce a cluster of 16 interconnected cystocytes. These clusters, or cysts, become surrounded by mesodermally derived follicle cells and develop into egg chambers at stage 1 . The stages of oogenesis represent a continuum of oocyte growth and maturation, as one member of the 16-cell cyst (the developing oocyte) grows at the expense of the other 15 germ-line cells ("nurse cells") contained within the cyst. During oocyte development, the nurse cells supply the ooplasm with cytosolic organelles and the maternally encoded transcripts required for early determinative events within the embryo. Morphologically, each ovary consists of an array of 15-20 ovarioles that contain developing oocytes arranged in order of increasing maturity (King 1970; Mahowald and Kambysellis 1980).

Digoxigenin-labeled DNA probes were used to study tud distribution in whole-mount ovaries, and ${ }^{35}$ S-labeled RNA probes (both sense and antisense) were used to probe sectioned ovaries. Using these techniques, we found that the tud transcript is first detectable in the germarium (see Fig. 5). The cystoblast cells, which are the direct products of division of the stem-line oogonia (region 1; King 1970), show no apparent staining with the tud probes. However, within the early cysts, or egg chambers, of region 2 (derived from incomplete divisions of the cystoblasts), one cystocyte appears to contain tud mRNA (see Fig. 5, arrowheads a and b). In later germarial cysts, this cell appears to be the most posterior cystocyte (see Fig. 5, arrowhead c, corresponding to germarium region 3). In stage 1 egg chambers, tud mRNA is clearly visible surrounding the oocyte nucleus (Fig. 5, arrowhead d). This staining pattern appears to persist at stage 2 . However, while the tud mRNA is still present in the oocyte cytoplasm at stage 3 , it no longer completely surrounds the oocyte nucleus. By stages 4-7, the tud mes- sage is found concentrated within the posterior half of the developing oocyte, with staining intensity reaching a maximum during stage 4 or 5 (Fig. $6 \mathrm{~B}$ ). Subsequently, the amount of tud mRNA appears to decrease steadily until stage 8 , when no signal above background can be detected within the egg chamber. By stage 10 , the nurse cells begin to produce large quantities of tud mRNA; however, no staining above background levels is visible in the oocyte (Fig. 6D). From late oogenesis throughout early embyrogenesis $(0-3 \mathrm{hr})$, the tud message remains undetectable but may be evenly distributed at a low level within the developing embryo. Our results are consistent with tud being required during an early oogenetic process for establishing the informational content of the posterior portion of the oocyte.

\section{Discussion}

In this report we present the initial molecular analysis of the tudor locus of $D$. melanogaster. On the basis of previous genetic analyses, tud mutations are strict, recessive, maternal-effect mutations. Regardless of their genotype, progeny of homozygous mutant tud females fail to form pole cells, and $\sim 40 \%$ die during embryogenesis, exhibiting severe abdominal segmentation defects (Boswell and Mahowald 1985b). In addition, the progeny of homozygous mutant tud females display an allelespecific reduction in the amount of assembled polar granule material. Thus, the two defects associated with tud mutations appear to result from aberrations in a process common to pole-cell determination and posterior pattern formation. The simplest explanation is that $\mathrm{tud}^{+}$is required for the proper establishment, localization, assembly, maintenance, and/or activity of the posterior organizing center.

The molecular analysis described herein is consistent with this view. While the tud transcript is expressed throughout the life cycle, it appears to be specifically localized during development of the female germ line. In the ovary, tud mRNA can be detected as early as the 
Figure 6. (A) Two wild-type ovarioles (whole mount) hybridized with digoxigenin-labeled tud CDNA probe ST58, showing labeling of the oocyte in stages 1-4. The anterior germaria $G$ are at the top, with egg chambers of stages $1-4$ extending posteriorly toward the bottom. The oocyte occupies a posterior position at the bottom of each egg chamber. Stages are numbered and labeling is at arrowheads. (B) Highmagnification view of oogenetic stage 5 from a wild-type ovariole prepared and hybridized as described in $A .(B-D)$ Anterior is to left. Note localization of the tud transcript to the posterior-most portion of the developing oocyte. $(C)$ Egg chambers at stages 6 (left) and 7 (right) of oogenesis. tud transcript label is still visible on the posterior side of the oocyte. $(D)$ Stage 10 egg chamber showing perinuclear staining in the nurse cells and no visible staining within the oocyte.

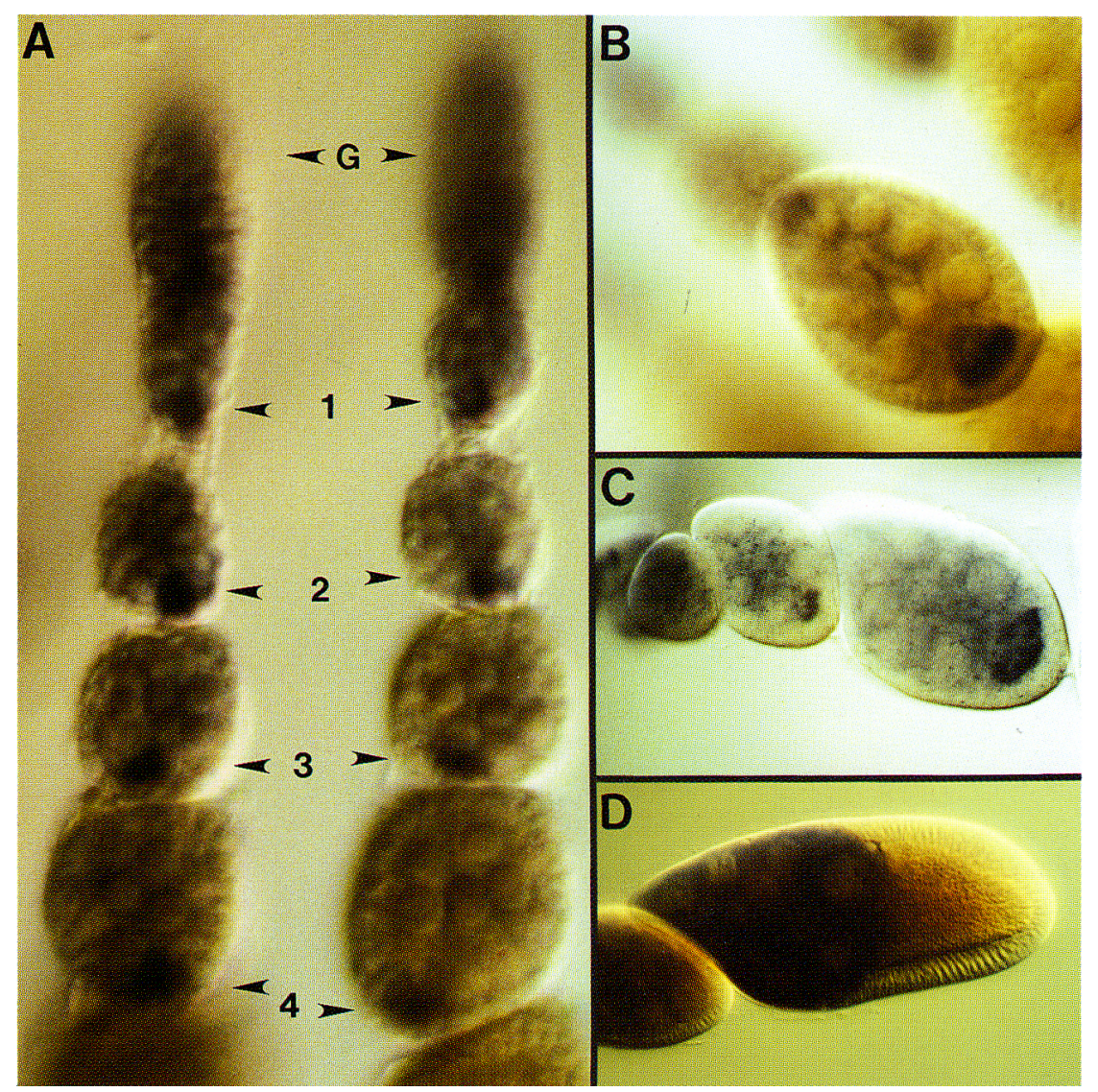

germarium stages within the cysts of 16 interconnected germ-line cells, which are precursors of the later oocytenurse cell complexes. The tud transcript appears to be present in one cell of each cyst, whereas the remaining visible cells, or cystocytes, are virtually free of staining. The cystocyte expressing tud mRNA is most likely the oocyte precursor, as it clearly occupies a posterior position within the later cysts, which subsequently form stage 1 egg chambers (see Fig. 5, arrowhead c). It is interesting to note that tud mRNA is present in one cystocyte even before the cyst has been rearranged to position the pro-oocyte at the posterior end. Although the mechanism of oocyte determination is not understood, it is thought to occur within germarium region 3 (Koch and King 1966; King 1970). At this point, the cystocyte destined to become the oocyte has moved from a more central location within the cyst in region 2 to the posterior end of the cyst. Whether the $t u d^{+}$gene product is required for this very early developmental event is not known, but the presence of tud mRNA at such early stages is intriguing.

Because the oocyte is transcriptionally inactive throughout most of oogenesis (King 1970), the nurse cells probably synthesize and export tud RNA during the earliest stages of oogenesis (stages 1-3). During the middle stages of oogenesis (stages 4-7), tud mRNA is localized within the posterior-most region of the developing oocyte. In many ovarioles examined by in situ hybridization to tud cDNAs, a distinctly labeled crescent shape is observed posterior to the oocyte nucleus (see Fig. $6 \mathrm{~B}, \mathrm{C})$. At stage 8 , little or no tud mRNA can be detected within the egg chamber. As oogenesis proceeds through stages 10-14, the nurse cells again begin to express tud mRNA and deposit it within the oocyte. However, the tud mRNA is not localized within the late oocyte at the time of egg deposition and cannot be detected above background staining in the egg cytoplasm. The transient localization of tud mRNA may play a role in the establishment of a functional posterior organizing center and/ or an active germ plasm during the early or middle stages of oogenesis. Unfortunately, the lack of temperaturesensitive alleles of tud does not allow us to determine whether this stage of oogenesis is a critical period for $\mathrm{tud}^{+}$synthesis and/or function. However, the production of antisera against tud protein is in progress and will allow us to determine the distribution of tud protein with far greater resolution. In any case, the tud transcript is present within the oocyte extremely early in oogenesis, perhaps from its outset. Similarly, the RNA product of the posterior group gene oskar has also been shown to be expressed in the presumptive oocyte at the earliest stages of its development in the germarium (Kim-Ha et al. 1991; Ephrussi et al. 1991). This transcript becomes localized to the posterior pole of the oocyte during mid- 
oogenesis. The vasa locus also encodes a transcript that is abundant in the germarium and nurse cells throughout the early stages of oogenesis. Although this RNA is not localized, the vasa protein is expressed from the outset of oogenesis and becomes tightly localized to the posterior pole around stage 10 (Hay et al. 1988; Lasko and Ashburner 1988). In addition, staufen RNA is expressed in the oocyte beginning at stage 3 , and its protein product is detectable concurrently or soon afterward (St. Johnston et al. 1991). Thus, in all cases analyzed to date, the gene products of the posterior group are expressed and/or localized very early in oogenesis.

The early expression of these genes raises questions about how early in oogenesis the posterior organizing center is formed. Illmensee et al. (1976) have demonstrated that polar plasm from stage 13 and stage 14 oocytes or from unfertilized eggs is capable of inducing germ-cell formation. However, although polar plasm from stages 10-12 appeared similar ultrastructurally to that of stages 13 and 14 (Mahowald 1962), it lacked activity in this assay. Thus, the germ plasm of the developing oocyte becomes fully competent between stages 12 and 13 of oogenesis. In contrast, oocytes and nurse cells from stage 10 egg chambers contain an activity capable of alleviating the abdominal defects associated with oskar mutations (Sander and Lehmann 1988). This posterior morphogenetic activity is distributed throughout the stage 10 oocyte-nurse cell complex but is apparently most abundant within the nurse cells. However, like the germ-cell determinants, this posterior activity is localized to the posterior pole of the egg by the end of oogenesis. How these two activities and their mecha$\mathrm{nism}(\mathrm{s})$ of localization are related remains unclear. Nonetheless, as Sander and Lehmann (1988) did not assay egg chambers earlier than stage 10 for posterior rescuing activity and as oskar, staufen, tud, and vasa are expressed very early in oogenesis, it is possible that the posterior morphogenetic activity becomes functional quite early during oogenesis.

In addition to the extremely early expression of tud, an additional unexpected result is our finding that the tud transcript is present throughout the Drosophila life cycle. If the $t u d^{+}$product is an essential component of polar granules, their assembly, or their stability, $t u d^{+}$ may be required throughout the life cycle to maintain the structural integrity of the polar granules /Mahowald 1971). Alternatively, $\mathrm{tud}^{+}$may be necessary for the maintenance and/or differentiation of the germ line during all developmental stages (see below). Nonetheless, with respect to its late expression, tud is similar to Toll and Bicaudal- $D$, other loci that are required maternally, yet are also expressed at later developmental stages (Hashimoto et al. 1988; Suter et al. 1989). The posterior group gene staufen has also been shown to be expressed throughout the life cycle (St. Johnston et al. 1991). Moreover, the early phenotype associated with tud mutations must be considered in light of the fact that we cannot be certain that any of the existing tud alleles are null. Females homozygous for the most severe alleles of Bicoidal-C, Bicoidal-D, a chromosomal deletion of vasa, or two alleles of nanos $\left(\operatorname{nos}^{R C}\right.$ and nos $\left.{ }^{R D}\right)$, fail to differentiate oocytes (Mohler and Wieschaus 1985; Lasko and Ashburner 1988; Lehmann and Nüsslein-Volhard 1991). Weaker alleles of these loci, however, allow the completion of oogenesis and, instead, result in embryonic pattern defects. These findings and the extremely early expression of $t u d$ suggest that the $t u d^{+}$gene product may also be required for oocyte differentiation. Thus, to clarify both the early and late function of tud, overlapping chromosomal deletions or known amorphic alleles must be studied.

Establishment of the informational content of the posterior pole of the Drosophila oocyte must involve an elaborate set of interactions. At least 10 maternal-effect loci, cappuccino, mago nashi, nanos, oskar, pumilio, spire, staufen, tud, valois, and vasa, are required for the normal development of the posterior of the Drosophila embryo (Boswell and Mahowald 1985b; Lehmann and Nüsslein-Volhard 1986; Schüpbach and Wieschaus 1986; Manseau and Schüpbach 1989b, Boswell et al. 1991). The molecular identity of the individual gene products encoded by the posterior-group loci should help clarify the nature of the instructive activity(ies) that these genes produce individually or collectively. However, with the exception of vasa, the posterior group gene products that have been examined at the molecular level loskar, staufen, tud) show no clear similarity to any proteins currently in the data bases, nor do they show any similarity to each other. Nontheless, through the continuing study of these gene products and their interactions, and the molecular cloning of the other members of the posterior group, significant insights into how positional information is generated at the posterior tip of the Drosophila oocyte should be provided in the near future.

\section{Materials and methods}

Fly strains and culturing

Most fly stocks used in this work have been described elsewhere (Boswell and Mahowald 1985b; O'Donnell et al. 1989). The tud $d^{5}$ allele was isolated in the same $\mathrm{X}$-ray mutagenesis that produced $t u d^{2}, t u d^{3}$, and $t u d^{4}$ (Boswell and Mahowald 1985b). $t u d^{B 46}$ is an $\mathrm{X}$-ray-induced allele isolated by Schüpbach and Wieschaus (1986). Except where noted, the wild-type stock in all experiments was Oregon-R. Flies were cultured in half-pint milk bottles or in 8 -dram vials on standard Drosophila medium at $25^{\circ} \mathrm{C}$.

\section{Chromosomal walk and genomic and cDNA libraries}

An EMBL4 recombinant phage library of $c n$ bw Drosophila genomic DNA, prepared according to Bender et al. (1983), and a Charon 4A library of Canton-S genomic DNA (Maniatis et al. 1978) were screened repeatedly during the chromosomal walk. The entry point was $\lambda 525$ (Levy et al. 1982), which hybridizes to the polytene interval $57 \mathrm{C}$. Restriction fragments of the distal and proximal ends of the recombinant phage inserts were isolated from low-melt agarose (BRL), labeled with ${ }^{32}$ P-labeled nucleotides, and used to reprobe the libraries. The direction of each step taken was determined by in situ hybridization of probes to polytene chromosomes of $\operatorname{In}(2 R) P u^{r 1} / O^{\prime}$ Donnell et al. 
1989). The chromosomal walk has been described in greater detail by McLean et al. (1990).

The cosmid library was constructed from a stock isogenized for all four chromosomes and was obtained from Tamkun (University of California at Santa Cruz). cDNAs were recovered from a 0 - to $24-\mathrm{hr}$ embryonic $\lambda \mathrm{gt} 1 \mathrm{l}$ library that was generated by oligo d $(T)$ primers (as described in Tamkun et al. 1991). In addition, a commercially available $\lambda$-ZAP embryonic cDNA library (which is a mixture of oligo $\mathrm{d}(\mathrm{T})$-primed and randomprimed cDNA) was obtained from Stratagene and screened to obtain the final piece of tud cDNA. All plaque and colony lifts were performed as described by Sambrook et al. (1989).

\section{In situ hybridization to polytene chromosomes and embryonic tissues}

Polytene chromosomes were obtained from salivary glands of third-instar larvae and were prepared by standard chromosome squash methods (Johnson-Schlitz and Lim 1987). In our earlier experiments, in situ hybridization was carried out with ${ }^{3} \mathrm{H}$-labeled probes according to the method of Johnson-Schlitz and Lim (1987). Subsequent experiments were performed with digoxigenin-labeled probes by using reagents available from Boehringer Mannheim Biochemicals. In these hybridizations, bound probe was detected by incubation of slides in an antidigoxigenin antibody conjugated to horseradish peroxidase, which was detected upon incubation of the slide in $\mathrm{H}_{2} \mathrm{O}_{2}$ and diaminobenzidine. This protocol was developed by Newmark, and will be described elsewhere (P. Newmark and R.E. Boswell, in prep.).

Whole-mount in situ hybridization to ovaries and embryos with digoxigenin-labeled probes (Boehringer Mannheim Biochemicals) was performed as described by Tautz and Pfeifle (1989) with the following modifications: Ovaries were dissected into either PBS plus $0.1 \%$ Tween 20 (PBT) or directly into fixative containing $0.1 \mathrm{M}$ HEPES $(\mathrm{pH} 6.9), 2 \mathrm{~mm} \mathrm{MgSO}_{4}, 1 \mathrm{~mm}$ EGTA, and $4 \%$ paraformaldehyde and fixed for $20 \mathrm{~min}$. The fixed ovaries were then washed three times in PBT, followed by a 3-min treatment in $50 \mu \mathrm{g} / \mathrm{ml}$ of proteinase $\mathrm{K}$ in PBT. Thereafter, the ovaries were treated according to the method of Tautz and Pfeifle (1989). This protocol was kindly provided by D. Marcey. As a control for the efficacy of this technique for detecting transcripts in late-stage egg chambers (stage 10-14), a digoxigenin labeled bicoid cDNA probe was hybridized to whole-mount ovaries. Labeling at the anterior of the developing oocyte was detectable through stage 13 . In addition to wholemount preparations, tissue sections $(10 \mu \mathrm{m})$ were prepared from paraffin-embedded ovaries and early embryos for hybridization with a ${ }^{35}$ S-labeled antisense RNA probe as described by Ingham et al. (1985). In all experiments, ${ }^{35} \mathrm{~S}$-labeled sense-strand probe were used as controls.

\section{Preparation and electrophoresis of RNA}

For embryo collections, adult flies of the appropriate genotype were placed in large plexiglass population cages into which a media tray (containing $15 \%$ unsulfured molasses and $1.5 \%$ agar) covered with yeast paste (baker's yeast plus water) was placed. The flies were then allowed to lay for a specific time interval, the media trays were removed, and the trays were incubated for the appropriate time interval at $25^{\circ} \mathrm{C}$. The embryos were rinsed from the tray with $\mathrm{H}_{2} \mathrm{O}$, washed several times in embryo wash buffer $\{0.4 \% \mathrm{NaCl}, 0.3 \%$ Triton $\mathrm{X}-100)$, and frozen in liquid nitrogen. Larvae and pupae were collected from half-pint milk bottles and harvested as described above.

RNA was isolated as described in Laughon et al. (1986).
Briefly, tissues were homogenized in $5 \mathrm{M}$ guanidine thiocyanate, $50 \mathrm{mM}$ Tris $\langle\mathrm{pH} \mathrm{7.5}\rangle, 20 \mathrm{mM} \beta$-mercaptoethanol, and $10 \mathrm{~mm}$ EDTA. Following a 10-min centrifugation at $16,000 \mathrm{~g}, 6 \mathrm{M} \mathrm{LiCl}$ was added to the supernatant until the final concentration of $\mathrm{LiCl}$ reached $3.3 \mathrm{M}$. After an overnight precipitation at $4^{\circ} \mathrm{C}$, the RNA was recovered by centrifugation at $16,000 \mathrm{~g}$ for $30 \mathrm{~min}$. The pellets were washed in $3 \mathrm{M} \mathrm{LiCl}, 4 \mathrm{M}$ urea, followed by another centrifugation at $16,000 \mathrm{~g}$ for $30 \mathrm{~min}$. The pellets were then drained well and thoroughly resuspended in TE $(10 \mathrm{~mm}$ Tris, $1 \mathrm{mM}$ EDTA/ plus $1 \%$ SDS. The dissolved RNA was then extracted with phenolchloroform $(1: 1)$ three times and with chloroform once. Following ethanol precipitation, the RNA was resuspended in nuclease-free $\mathrm{H}_{2} \mathrm{O}$ and stored at $-80^{\circ} \mathrm{C}$. Poly $\mid \mathrm{A})^{+}$RNA was then prepared according to the procedure described by Hynes et al. (1987).

RNA was separated by electrophoresis on $1 \%$ agarose-formaldehyde gels in a MOPS buffer (Larkin and Woolford 1983). RNA size standards (Bethesda Research Laboratories) were used for molecular weight determinations. Following electrophoresis, gels were rinsed twice in $10 \times$ SSC and transferred for $>10 \mathrm{hr}$ to Zetabind (CUNO Corp.) in $20 \times$ SSC.

\section{Nucleic acid hybridizations}

${ }^{32}$ P-Labeled DNA probes were prepared using the random hexamer primer technique of Feinberg and Vogelstein (1983). All DNA-DNA hybridizations (Southern blots, colony-filter hybridizations, and plaque lifts| were prehybridized and hybridized at $65^{\circ} \mathrm{C}$ in a buffer containing $6 \times$ SSC, $0.5 \%$ SDS, $5 \times$ Denhardt's solution, and $0.5 \mathrm{mg} / \mathrm{ml}$ of salmon sperm DNA. Following hybridization, the filters were washed as described by Sambrook (1989). Northern blots were prehybridized for $>8 \mathrm{hr}$ at $45^{\circ} \mathrm{C}$ in $50 \%$ formamide, $4 \times$ SSC, $50 \mathrm{~mm}$ potassium phosphate ( $\mathrm{pH} 6.5$ ), $5 \times$ Denhardt's solution, and $0.4 \mathrm{mg} / \mathrm{ml}$ of salmon sperm DNA; hybridized overnight at $45^{\circ} \mathrm{C}$ in prehybridization solution that also contained $10 \%$ dextran sulfate and $0.05 \% \mathrm{SDS}$; and washed $4 \times 30 \mathrm{~min}$ in $0.1 \times \mathrm{SSC}, 0.5 \% \mathrm{SDS}$ at $68^{\circ} \mathrm{C}$. Finally, single-stranded riboprobes (Melton et al. 1984) were used to determine the direction of tud transcription and were used in some in situ hybridizations. These probes were prepared with reagents and protocols obtained from Promega Corporation.

\section{DNA sequence analysis}

The complete sequence of both strands of the transcribed regions of tud was determined as follows: Nested deletions were produced by treatment of appropriately digested clones with exonuclease III (Henikoff 1987) with reagents and detailed protocols from Promega Corporation. The serially deleted subclones were sequenced by the dideoxy method (Sanger et al. 1977) with the 7-deaza-dGTP reagent kit for DNA sequencing with Sequenase (U.S. Biochemical Corporation). Two kilobases of one strand of the ST3 cDNA and one strand of the 9Al cDNA were sequenced by using synthetic primers purchased from Operon Corporation.

The sequence data base search was done with the TFASTA and BLAST programs (Lipman and Pearson 1985; Pearson and Lipman 1988; Altschul et al. 1990). In addition, the tud protein sequence was examined for structural motifs shared between functionally related polypeptides as reported in Bairoch (1989).

\section{Acknowledgments}

We are grateful to Dr. Janis O'Donnell for providing us with the tud cDNA clone JO, and to Dr. John Bermingham for collabo- 
rating on the preparation of adult male and female RNA. In addition, we thank Drs. Michael Klymkowsky and Gary Stormo for critical reading of the manuscript and to Dr. Kent McDonald for photographic assistance. Finally, special gratitude goes to Dr. John Tamkun for many valuable suggestions and for teaching G.S.G. numerous techniques. This research was supported by grants to R.E.B. from the National Institutes of Health (R01GM36937), the National Science Foundation (DCB-8905118), and the March of Dimes Foundation (basic grant 1-1161). R.E.B. is also a recipient of an award from the PEW Scholars Program. We also thank the W.M. Keck Foundation for their generous support of RNA science on the Boulder campus.

The publication costs of this article were defrayed in part by payment of page charges. This article must therefore be hereby marked "advertisement" in accordance with 18 USC section 1734 solely to indicate this fact.

\section{Note added in proof}

Sequence data described in this paper have been submitted to EMBL/GenBank under accession number X62420.

\section{References}

Altschul, S.F., W. Gish, W. Miller, E.W. Myers, and D.J. Lipman. 1990. Basic local alignment search tool. I. Mol. Biol. 215: 403-410.

Anderson, K.V. 1987. Dorsal-ventral embryonic pattern genes in Drosophila. Trends Genet. 3: 91-97.

Bairoch, A. 1989. PROSITE: A dictionary of protein sites and patterns, Release 4. Université of Geneve and EMBL, Geneva.

Bender, W., M. Akam, F. Karch, P.A. Beachy, M. Peifer, P. Spierer, E.B.Lewis, and D.S. Hogness. 1983. Molecular genetics of the Bithorax Complex of Drosophila melanogaster. Science 221: 23-29.

Boswell, R.E. and A.P. Mahowald. 1985a. Cytoplasmic determinants in embryogenesis. In Comprehensive insect physiology, biochemistry and pharmacology (ed. G.A. Kerkut and L.I. Gilbert); pp. 387-405. Pergamon Press, Oxford.

- 1985b. tudor, a gene required for assembly of the germ plasm in Drosophila melanogaster. Cell 43: 97-104.

Boswell, R.E., M.E. Prout, and J.C. Steichen. 1991. Mutations in a newly identified Drosophila melanogaster gene, mago nashi, disrupt germ cell formation and result in the formation of mirror-image symmetrical double abdomen embryos. Development 113: 373-384.

Cavener, D.R. 1987. Comparison of the consensus sequence flanking translational start sites in Drosophila and vertebrates. Nucleic Acids Res. 15: 1353-1361.

Davidson, E.H. 1986. Gene activity in early development, 3rd ed. Academic Press, New York.

Driever, W., V. Siegel and C. Nüsslein-Volhard. 1990. Autonomous determination of anterior structures in the early Drosophila embryo by bicoid morphogen. Development 109: 811-820.

Eddy, E.M. 1975. Germ plasm and differentiation of the germ line. Int. Rev. Cytol. 43: 229-280.

Ephrussi, A., L.K. Dickinson, and R. Lehmann. 1991. oskar organizes the germ plasm and directs localization of the posterior determinant nanos. Cell 66: 37-50.

Feinberg, A.P. and B. Vogelstein. 1983. A technique for radiolabeling DNA restriction endonuclease fragments to high specific activity. Anal. Biochem. 132: 6-13.

Frohnhöfer, H.G. and C. Nüsslein-Volhard. 1986. The organiza- tion of anterior pattern in the Drosophila embryo by the maternal gene bicoid. Nature 324: 120-125.

Frohnhöfer, H.G., R. Lehmann, and C. Nüsslein-Volhard. 1986. Manipulating the anteroposterior pattern of the Drosophila embryo. J. Embryol. Exp. Morphol. 97:169-179.

Fyrberg, E.A., K.L. Kindle, N. Davidson, and A. Sodja. 1980. The actin genes of Drosophila: A dispersed multigene family. Cell 19: 365-378.

Hashimoto, C., K.L. Hudson, and K.V. Anderson. 1988. The Toll gene of Drosophila, required for dorsal-ventral embryonic polarity, appears to encode a transmembrane protein. Cell 52: 269-279.

Hay, B., L. Ackerman, S. Barbel, L.Y. Jan, and Y.N. Jan. 1988. Identification of a component of Drosophila polar granules. Development 103: 625-640.

Henikoff, S. 1987. Unidirectional digestion with exonuclease III in DNA sequence analysis. Methods Enzymol. 155: 156165.

Hynes, R., J.E. Schwarzbauer, and J.W. Tamkun. 1987. Isolation and analysis of cDNA and genomic clones of fibronectin and its receptor. Methods Enzymol. 144: 447-463.

Illmensee, K. and A.P. Mahowald. 1974. Transplantation of posterior polar plasm in Drosophila. Induction of germ cells at the anterior pole of the egg. Proc. Natl. Acad. Sci. 71: 10161020 .

- 1976. The autonomous function of germ plasm in a somatic region of the Drosophila egg. Exp. Cell Res. 97: 127140.

Illmensee, K., A.P. Mahowald, and M.R. Loomis. 1976. The otogeny of germ plasm during oogenesis in Drosophila. Dev. Biol. 49: 40-65.

Ingham, P.W., K.R. Howard, and D. Ish-Horowicz. 1985. Transcription pattern of the Drosophila segmentation gene hairy. Nature 318: 439-445.

Johnson-Schlitz, D. and J.K. Lim. 1987. Cytogenetics of Notch mutations arising in the unstable $\mathrm{X}$ chromosome $U c$ of Drosophilamelanogaster. Genetics 115: 701-709.

Kim-Ha, J., J.L. Smith, and P.M. Macdonald. 1991. oskar mRNA is localized to the posterior pole of the Drosophila oocyte. Cell 66: 23-35.

King, R.C. 1970. Ovarian development in Drosophila melanogaster. Academic Press, New York.

Koch, E.A. and R.C. King. 1966. The origin and early differentiation of the egg chamber of Drosophila melanogaster. I. Morphol. 119: 283-304.

Larkin, J.C. and J.L. Woolford. 1983. Molecular cloning and analysis of the $C R Y 1$ gene: A yeast ribosomal protein gene. Nucleic Acids Res. 11: 403-420.

Lasko, P.F. and M. Ashburner. 1988. The product of the Drosophilagene vasa is very similar to eukaryotic initiation factor4A. Nature 335: 611-617.

Laughon, A., A.M. Boulet, J.R.J. Bermingham, R.A. Laymon, and M.P. Scott. 1986. Structure of transcripts from the homeotic Antennapedia gene of Drosophila melanogaster: Two promoters control the major protein-coding region. Mol. Cell. Biol. 6: 4676-4689.

Lehmann, R. and C. Nüsslein-Volhard. 1986. Abdominal segmentation, pole cell formation, and embryonic polarity require the localized activity of oskar, a maternal gene in Drosophila. Cell 47: 141-152.

-1987. Involvement of the pumilio gene in the transport of an abdominal signal in the Drosophila embryo. Nature 329: $167-170$.

- 1991. The maternal gene nanos has a central role in posterior pattern formation of the Drosophila embryo. De velopment 112: 679-691. 
Levy, L.S., R. Ganguly, N. Ganguly, and J.E. Manning. 1982. The selection, expression, and organization of a set of head-specific genes in Drosophila. Dev. Biol. 94: 451-464.

Lipman, D.J. and W.R. Pearson. 1985. Rapid and sensitive protein similarity searches. Science 227: 1435-1441.

Mahowald, A.P. 1962. Fine structure of pole cells and polar granules in Drosophila melanogaster. J. Exp. Zool. 151: 201215.

1971. Polar granules of Drosophila. III. The continuity of polar granules during the life cycle of Drosophila. I. Exp. Zool. 176: 329-344.

1977. The germ plasm of Drosophila: An experimental system for the analysis of germ cell determination. Am. Zool. 17: 551-563.

Mahowald, A.P. and M.P. Kambysellis. 1980. Oogenesis. In The genetics and biology of Drosophila (ed. M. Ashburner and T.R.F. Wright). pp. 141-224. Academic Press, New York.

Maniatis, T., R.C. Hardison, E. Lacy, J. Lauer, C. O'Connell, D. Quon, G.K. Sim, and A. Efstradiatis. 1978. The isolation of structural genes from libraries of eucaryotic DNA. Cell 15: $687-701$.

Manseau, L.J. and T. Schüpbach. 1989a. The egg came first, of course! Anterior-posterior pattern formation in Drosophila embryogenesis and oogenesis. Trends Genet. 5: 400-405.

- 1989b. cappuccino and spire: Two unique maternal-effect loci required for both the anteroposterior and dorsoventral patterns of the Drosophila embryo. Genes \& Dev. 3: $1437-1452$.

McLean, J.R., R. Boswell, and J. O'Donnell. 1990. Cloning and molecular characterization of a metabolic gene with developmentalfunctions in Drosophila.1. Analysis of the head function of Punch. Genetics 126: 1007-1019.

Melton, D.A., P.A. Krieg, M.R. Rebagliate, T. Maniatis, K. Zinn, and M.R. Green. 1984. Efficient in vitro synthesis of biologically active RNA and RNA hybridization probes from plasmids containing a bacteriophage SP6 promoter. Nucleic Acids Res. 12: 7035-7070.

Mohler, J. and E.F. Wieschaus. 1985. Bicaudal mutations of Drosophila melanogaster: Alteration of blastoderm cell fate. Cold Spring Harbor Symp. Quant. Biol. 50: 105-111.

Nüsslein-Volhard, C., H.G. Frohnhöfer and R. Lehmann. 1987. Determination of anteroposterior polarity in Drosophila. Science 238: 1675-1681.

O'Donnell, J., R. Boswell, T. Reynolds, and W. Mackay. 1989. A cytogenetic analysis of the Punch-tudor region of chromosome 2R in Drosophila melanogaster. Genetics 121:273280.

Pearson, W.R. and D.J. Lipman. 1988. Improved tools for biological sequence comparison. Proc. Natl. Acad. Sci. 85: 2444-2448.

Roth, S., D. Stein, and C. Nüsslein-Volhard. 1989. A gradient of nuclear localization of the dorsal protein determines dorsoventral pattern in the Drosophila embryo. Cell 59: 11891202.

Rushlow, C.A., K.Y. Han, J.L. Manley, and M. Levine. 1989. The gradeddistribution of the dorsal morphogen is initiated by selective nuclear transport in Drosophila. Cell 59: 11651177.

St. Johnston, D., D. Beuchle, and C. Nüsslein-Volhard. 1991. staufen, a gene required to localize maternal RNAs in the Drosophila egg. Cell 66: 51-63.

Sambrook, J., E.F. Fritsch, and T. Maniatis. 1989. Molecular cloning. A laboratory manual, 2nd ed. Cold Spring Harbor Laboratory Press, New York.

Sander, K. and R. Lehmann. 1988. Drosophila nurse cells produce a posterior signal required for embryonic segmentation and polarity. Nature 335: 68-70.

Sanger, F., S. Nicklen, and A.R. Coulson. 1977. DNA sequencing with chain-terminating inhibitors. Proc. Natl. Acad. Sci. 74: 5463-5467.

Schüpbach, T. and E. Wieschaus. 1986. Maternal-effect mutationsaltering the anterior-posterior pattern of the Drosophila embryo. Wilhelm Roux's Arch. Dev. Biol. 195: 302-317.

Smith, L.D. and M.A. Williams. 1975. Germ plasm and determination of the primordial germ cells. In The developmental biology of reproduction (ed. C. L. Markert and J. Papaconstantinoul, pp. 3-24. Academic Press, London and New York.

Smoller, D., C. Friedel, A. Schmid, D. Bettler, L. Lam, and B. Yedbovnick. 1990. The Drosophila neurogenic locus mastermind encodes a nuclear protein unusually rich in amino acid homopolymers. Genes \& Dev. 4: 1688-1700.

Steward, R. 1989. Relocalization of the dorsal protein from the cytoplasm to the nucleus correlates with its function. Cell 59: 1179-1188.

Strome, S. and W.B. Wood. 1981. Immunofluorescense visualization of germ-line specific cytoplasmic granules in embryos, larvae and adults of Caenorhabditis elegans. Proc. Natl. Acad. Sci. 79: 1558-1562.

Suter, B., L.M. Romberg, and R. Steward. 1989. Bicaudal-D, a Drosophila gene involved in developmental asymmetry: Localized transcript accumulation in ovaries and sequence similarity to myosin heavy chain tail domains. Genes \&) Dev. 3: 1957-1968.

Tamkun, J.W., R.A. Kahn, M. Kissinger, B.J. Brizuela, C. Rulka, M.P. Scott, and J.A. Kennison. 1991. The arflike gene encodes an essential GTP-binding protein in Drosophila. Proc. Natl. Acad. Sci. 88: 3120-3124.

Tautz, D. and C. Pfeifle. 1989. A non-radioactive in situ hybridization method for the localization of specific RNAs in Drosophila embryosreveals translational control of the segmentation gene hunchback. Chromosoma 98: 81-85.

Tobin, S.L., E. Zulauf, F. Sanchez, E.A. Craig, and B.J. McCarthy. 1980. Multiple actin-related sequences in the Drosophila melanogaster genome. Cell 19: 121-131. 


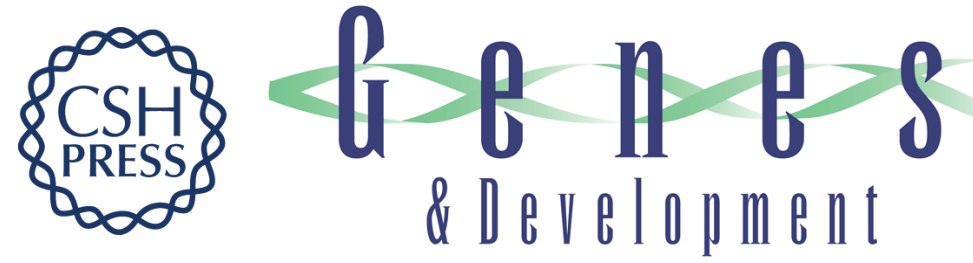

\section{tudor, a posterior-group gene of Drosophila melanogaster, encodes a novel protein and an mRNA localized during mid-oogenesis.}

G S Golumbeski, A Bardsley, F Tax, et al.

Genes Dev. 1991, 5:

Access the most recent version at doi:10.1101/gad.5.11.2060

References This article cites 57 articles, 19 of which can be accessed free at:

http://genesdev.cshlp.org/content/5/11/2060.full.html\#ref-list-1

License

Email Alerting

Service

Receive free email alerts when new articles cite this article - sign up in the box at the top right corner of the article or click here.

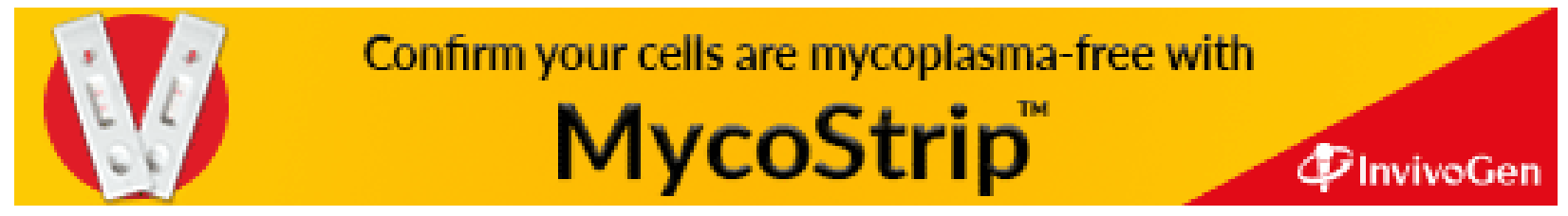

